\section{(A) OPEN ACCESS}

\title{
Glucocorticoids induce senescence in primary human tenocytes by inhibition of sirtuin 1 and activation of the p53/p21 pathway: in vivo and in vitro evidence
}

\author{
Raewyn C Poulsen, Anna C Watts, Richard J Murphy, Sarah J Snelling, Andrew J Carr, \\ Philippa A Hulley
}

\begin{abstract}
Handling editor Tore K Kvien
- Additional material is published online only. To view please visit the journal online (http://dx.doi.org/10.1136/ annrheumdis-2012-203146)

Nuffield Department of Orthopaedics, Rheumatology and Musculoskeletal Sciences, Botnar Research Centre, University of Oxford, Oxford, UK
\end{abstract}

\section{Correspondence to}

Dr Raewyn C Poulsen, Nuffield Department of Orthopaedics, Rheumatology and Musculoskeletal Sciences, Botnar Research Centre, University of Oxford, Oxford OX3 7D, UK; raewyn.poulsen@ ndorms.ox.ac.uk

Accepted 7 May 2013 Published Online First 1 June 2013
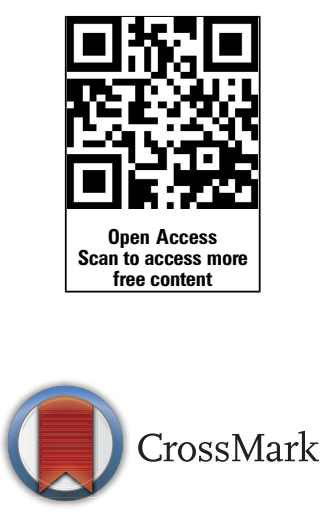

To cite: Poulsen $\mathrm{RC}$ Watts AC, Murphy RJ, et al. Ann Rheum Dis

2014;73:1405-1413.

\section{ABSTRACT}

Cellular senescence is an irreversible side effect of some pharmaceuticals which can contribute to tissue degeneration.

Objective To determine whether pharmaceutical glucocorticoids induce senescence in tenocytes. Methods Features of senescence ( $\beta$-galactosidase activity at pH 6 (SA- $\beta$-gal) and active mammalian/ mechanistic target of rapamycin (mTOR) in cell cycle arrest) as well as the activity of the two main pathways leading to cell senescence were examined in glucocorticoid-treated primary human tenocytes. Evidence of senescence-inducing pathway induction in vivo was obtained using immunohistochemistry on tendon biopsy specimens taken before and 7 weeks after subacromial Depo-Medrone injection.

Results Dexamethasone treatment of tenocytes resulted in an increased percentage of SA- $\beta$ gal-positive cells. Levels of phosphorylated p70S6K did not decrease with glucocorticoid treatment indicating mTOR remained active. Increased levels of acetylated p53 as well as increased RNA levels of its pro-senescence effector p21 were evident in dexamethasone-treated tenocytes. Levels of the p53 deacetylase sirtuin 1 were lower in dexamethasone-treated cells compared with controls. Knockdown of p53 or inhibition of p53 activity prevented dexamethasone-induced senescence. Activation of sirtuin 1 either by exogenous overexpression or by treatment with resveratrol or low glucose prevented dexamethasone-induced senescence. Immunohistochemical analysis of tendon biopsies taken before and after glucocorticoid injection revealed a significant increase in the percentage of p53-positive cells $(p=0.03)$. The percentage of $p 21$-positive cells also tended to be higher post-injection $(p=0.06)$ suggesting glucocorticoids activate the p53/p21 senescenceinducing pathway in vivo as well as in vitro.

Conclusion As cell senescence is irreversible in vivo, glucocorticoid-induced senescence may result in longterm degenerative changes in tendon tissue.

\section{INTRODUCTION}

Musculoskeletal pain is one of the most common complaints of patients seeking general practitioner consultations. Chronic musculoskeletal pain arising from conditions such as arthritis and rotator cuff disease is often particularly difficult to treat. When oral analgesics prove ineffective, local injection with synthetic glucocorticoids is frequently used. In the UK, over 500000 intra-articular glucocorticoid injections are administered per year in the primary care setting alone. ${ }^{1}$

Glucocorticoids are used to treat pathologies affecting a range of different tissues. Despite the prevalence of glucocorticoid use, there is concern glucocorticoids may have unwanted side effects. ${ }^{2-6}$ Glucocorticoids have been shown to induce cell cycle arrest and inhibit normal cell activity. ${ }^{7-10}$ However, it is not known whether these are transient effects quickly reversed once the glucocorticoid has dissipated from the tissue or whether the effects are more persistent.

Cell cycle arrest can lead to either quiescence or senescence. Quiescence is a reversible process leading to a transient state of cell dormancy. Senescence in contrast results in an irreversible change in normal cell activity. First observed in aged cells, cell senescence can also occur in young cells usually as a consequence of activation of either/both of the p38/ $\mathrm{p} 16^{\mathrm{INK} 4 \mathrm{a}}$ or $\mathrm{p} 53 / \mathrm{p} 21^{\text {cip }}$ stress-responsive signalling pathways. Senescent cells are hypersecretory, producing abnormal extracellular matrix as well as growth factors, cytokines and matrix-degrading enzymes. ${ }^{11}{ }^{12}$ Many of these molecules are implicated in the pathogenesis of tendinopathy. ${ }^{13}{ }^{14}$ Determining whether quiescence or senescence prevails following glucocorticoid treatment is therefore important for understanding the implications of glucocorticoid-induced cell cycle arrest.

Whether cell cycle arrest leads to quiescence or senescence hinges on the activity of mammalian/ mechanistic target of rapamycin (mTOR). ${ }^{15} \quad 16$ mTOR is the catalytic subunit of two enzyme complexes: mTORC1 and mTORC2. mTORC1 drives both cell proliferation and cell growth in part by phosphorylating the ribosomal protein S6 kinases. ${ }^{17-19}$ In quiescent cells, mTOR is inactive. However in senescent cells, mTOR remains active. ${ }^{15}$ As senescent cells are in cycle arrest, they are impervious to the pro-proliferative effects of $\mathrm{mTOR}$ but are responsive to its growth stimulatory effects. As a result, senescent cells continue to grow and appear enlarged compared with their nonsenescent counterparts. ${ }^{20}$ Senescence-associated hypertrophy leads to increased lysosomal number and activity. ${ }^{21}$ It is this feature which is exploited in one of the most widely used assays to identify 
senescence-the activity of lysosomal $\beta$-galactosidase $\left(\mathrm{SA}-\beta\right.$-gal). ${ }^{22}$

The aims of the present study were to determine whether glucocorticoids induce quiescence or senescence in tenocytes in vitro, to identify the signalling pathways controlling this cell fate decision and to confirm whether the same cell phenotype results in tendon tissue following local injection with glucocorticoids.

\section{METHODS}

\section{Cell culture}

Human tenocytes were obtained by explant culture of hamstring tendon used in repair of ruptured anterior cruciate ligament as previously described. ${ }^{10}$ Osteoblasts were obtained by explant culture of bone chips from osteoarthritic patients and chondrocytes by collagenase digestion of osteoarthritic knee cartilage. For more details, refer online supplementary information.

\section{RNAi-mediated gene silencing}

Gene knockdown was achieved using lipofectamine RNAimax (Life Technologies, Paisley, UK) following the manufacturer's instructions (for details refer online supplementary information). The transfection protocol did not alter cellular response to dexamethasone treatment (see online supplementary figure S1).

\section{Adenoviral-mediated gene transduction}

Tenocytes were infected with either a sirtuin 1 (adSirt1) or a green fluorescent protein (adGFP) adenoviral vector (refer online supplementary information for details) at MOI35 using Xtremegene HP (Roche Diagnostics Ltd, Burgess Hill, UK) following the manufacturer's instructions. The transduction protocol did not alter cellular response to dexamethasone treatment (see online supplementary figure S1).

\section{Western blotting}

Cells were sonicated in standard lysis buffer containing protease and phosphatase inhibitors. Western blots were carried out according to standard protocols ${ }^{10}$ and proteins visualised using Pierce WestDura detection reagents (ThermoScientific, Rockford, Illinois, USA) using a Chemi Doc-It Imaging System with Biochemi HR camera (UVP, Upland, California, USA).

\section{Real-time reverse transcription polymerase chain reaction (RT-PCR)}

cDNA was prepared using a cells-to-cDNA kit as per the manufacturer's instructions (Ambion, Austin, Texas, USA). Samples without reverse transcriptase and no-template controls served as negative controls. Real-time quantitative polymerase chain reaction (qPCR) reactions were performed using a Corbett Rotor-Gene 3000, a QuantiTect SYBR Green PCR kit and QuantiTect Primer Assays (Qiagen, Crawley, UK). All samples were run in duplicate with a coefficient of variation between duplicates of $<1.0$ cycle. Analysis was carried out using the delta-delta cT method. ${ }^{23}$

\section{Senescence-associated $\beta$-galactosidase staining}

SA- $\beta-$ Gal activity was determined as previously described ${ }^{22}$ with minor modifications (see online supplementary information). Cells were visualised using a BX40 Olympus microscope with a DP70 camera (Olympus Life Science Europa Gmbh, Hamburg, Germany) with Safranin $\mathrm{O}$ as a counterstain. The number of $\beta$-galactosidase-positive cells and the total number of cells were counted in 10 fields of view $(100 \times)$ per sample.

\section{Pre-glucocorticoid and post-glucocorticoid injection tendon biopsies}

Ethical approval for the study was granted by the local research ethics committee (Oxfordshire REC B, ref: 09/H0605/111). Supraspinatus tendon biopsies were taken from five patients undergoing subacromial glucocorticoid injection for rotator cuff tendinopathy (refer table 1 for patient details). All patients had subacromial impingement pain, 'painful arc' and positive Hawkins' and Jobe's tests. ${ }^{24-26}$ Structural integrity of the rotator cuff was assessed by ultrasound. Exclusion criteria were fullthickness rotator cuff tear, previous significant shoulder trauma, systemic steroid use or tobacco smoking, previous or current shoulder problems other than rotator cuff tendinopathy, previous shoulder surgery, local glucocorticoid injections in the last 6 weeks or a significant systemic medical condition, for example, diabetes or a rheumatological condition. Bonar scores as described in ref. [27] were used to compare the degree of tendinopathy pre-glucocorticoid and post-glucocorticoid injection (table 1).

Longitudinal tendon biopsies were taken at a consistent location $5-10 \mathrm{~mm}$ posterior to the rotator interval under ultrasound guidance using a $14 \mathrm{G}$ spring-loaded core biopsy needle. Biopsies were taken immediately prior to and 7 weeks following ultrasoundguided injection of $40 \mathrm{mg}$ Depo-Medrone into the subacromial bursa. Biopsies were immediately placed into $10 \%$ buffered formalin, subsequently wax embedded and sectioned $(5 \mu \mathrm{m})$.

\section{Immunohistochemistry}

Immunohistochemistry was performed using an EnVision FLEX High-pH kit and an AutostainerLink48 as per the manufacturer's instructions (Dako, Ely, UK). p53 and p21 antibodies (refer online supplementary information) were used at dilutions of $1: 50$ and $1: 100$, respectively.

\section{Statistical analysis}

All in vitro experiments were repeated at least three times using different tissue donors for each experimental replicate. The results for in vitro experiments were analysed by one-way analysis of variance (ANOVA) with post hoc Tukey test or by t test if only two conditions were being tested. Data obtained from immunohistochemistry on patient specimens were analysed using the non-parametric Wilcoxon matched pairs $t$ test. All data were analysed using Prism V.5.0b (GraphPad Software, La Jolla, California, USA). p Value $\leq 0.05$ was considered statistically significant. The results are expressed as mean $\pm S D$.

\section{RESULTS}

Glucocorticoid treatment results in cell morphological changes consistent with a senescent phenotype

To treat tendinopathy, typically $40 \mathrm{mg}$ of glucocorticoid suspended in 1-10 $\mathrm{ml}$ of saline is injected into the bursa (equating

Table 1 Pre-glucocorticoid and post-glucocorticoid injection biopsy study patient details

\begin{tabular}{|c|c|c|c|c|}
\hline \multirow[b]{2}{*}{ Sex } & \multirow[b]{2}{*}{ Age } & \multirow{2}{*}{$\begin{array}{l}\text { Previous glucocorticoid } \\
\text { injections }\end{array}$} & \multicolumn{2}{|l|}{ Bonar score* } \\
\hline & & & Pre-injection & Post-injection \\
\hline Female & 36 & 0 & 5 & 6 \\
\hline Female & 57 & 0 & 6 & 5 \\
\hline Female & 62 & 1 & 7 & 7 \\
\hline Female & 65 & 0 & 7 & 8 \\
\hline Male & 54 & 1 & 7 & 7 \\
\hline
\end{tabular}


to $10-100 \mathrm{mM}$ ). The glucocorticoid concentration to which tenocytes are exposed following local injection is difficult to assess and will depend on the perfusibility of the tendon tissue and the proximity of the tenocyte to the injection site. Following systemic administration, circulating glucocorticoid concentrations of approximately $1 \mu \mathrm{M}$ have been observed. ${ }^{28}$

As an initial screen, we treated tenocytes with $1 \mu \mathrm{M}$ dexamethasone. By 48 and $72 \mathrm{~h}$ post-treatment, a significant increase in the percentage of SA- $\beta$-gal-positive cells was observed (figure $1 \mathrm{~A}$, see online supplementary figure S2).
In order to determine the effect of lower glucocorticoid doses, we treated tenocytes with $50 \mathrm{nM}, 150 \mathrm{nM}, 350 \mathrm{nM}$ or $1 \mu \mathrm{M}$ dex for 3 days. We found treatment with any of the concentrations tested resulted in an increased percentage of SA- $\beta$-gal-positive cells (figure 1B). Prolonged treatment for 7 days did not result in a further increase in the percentage of SA- $\beta$-gal-positive cells (figure 1C). Co-treatment with the glucocorticoid receptor (GR) antagonist RU486 $(80 \mathrm{nM})$ for $72 \mathrm{~h}$ prevented the dex-induced increase in the percentage of SA- $\beta$-gal-positive cells, albeit more effectively, following $50 \mathrm{nM}$ rather than $1 \mu \mathrm{M}$ dexamethasone treatment (figure 1D).
Figure 1 Glucocorticoids cause morphological and functional changes in tenocytes consistent with a senescent phenotype. (A) The percentage of SA- $\beta$ gal-positive cells was significantly higher 48 and $72 \mathrm{~h}$ post-treatment of primary human tenocytes with $1 \mu \mathrm{M}$ dexamethasone compared with tenocytes treated with ethanol (carrier) alone. There was no evidence of a dose effect of dexamethasone treatment on the percentage of SA- $\beta$ gal-positive cells following (B) 3 days or (C) 7 days of treatment $(n=3)$ compared with cells treated with the corresponding amount of ethanol carrier. (D) The percentage of SA- $\beta$ gal-positive cells was significantly lower in cells treated for $72 \mathrm{~h}$ with dexamethasone $(50 \mathrm{nM}$ or $1 \mu \mathrm{M})$ and the glucocorticoid receptor (GR) antagonist RU486 (80 nM) compared with cells treated with dexamethasone alone, $n=3$.

(E) Western blot showing levels of phosphorylated and total p70 S6 kinase, a substrate of the mTORC1 complex and an indicator of mTORC1 activity 24,48 and $72 \mathrm{~h}$ post-dexamethasone (dex) treatment $(1 \mu \mathrm{M})$ in primary human tenocytes. Western blots were conducted on protein lysates from cells obtained from three different tissue donors. Blot shown is representative of all experiments. (F) No difference in RNA levels of either of the two p70 S6 kinase subunits ( $p 7056 \mathrm{KB1}$ and p70S6KB2) was evident in tenocytes treated with dexamethasone $(1 \mu \mathrm{M})$ compared with ethanol-treated controls. (G) A significantly lower percentage of SA- $\beta$ gal-positive cells was evident in cells treated for $72 \mathrm{~h}$ with dexamethasone (50 nM or $1 \mu \mathrm{M})$ and the mammalian/mechanistic target of rapamycin (mTOR) inhibitor rapamycin (rapa) compared with cells treated with dexamethasone alone, $\mathrm{n}=3$. Statistically significant differences between ethanol (EtOH) and dexamethasone (Dex) and between dexamethasone with and without either the GR inhibitor (RU486) or mTOR inhibitor (rapamycin) are indicated by * $(p<0.05)$.
A

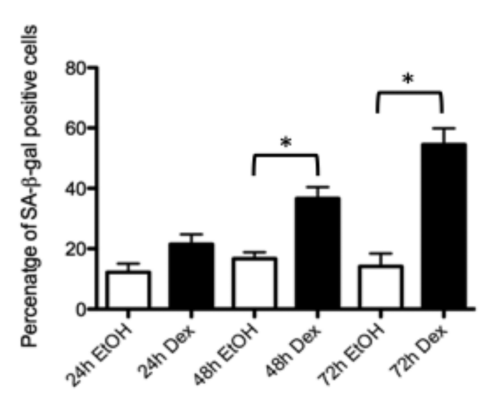

C

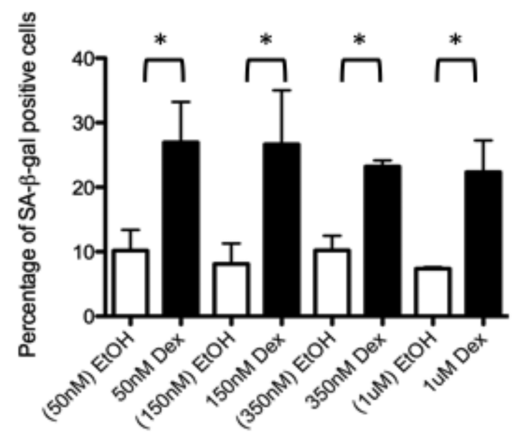

E

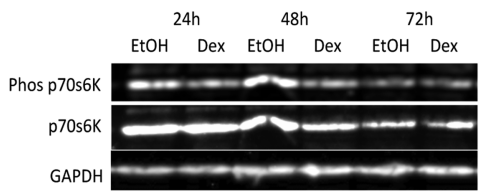

B

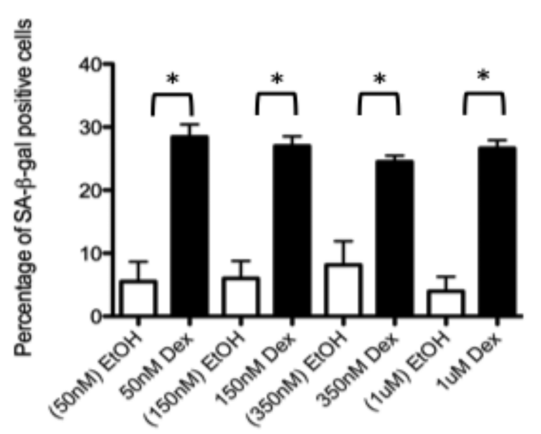

D
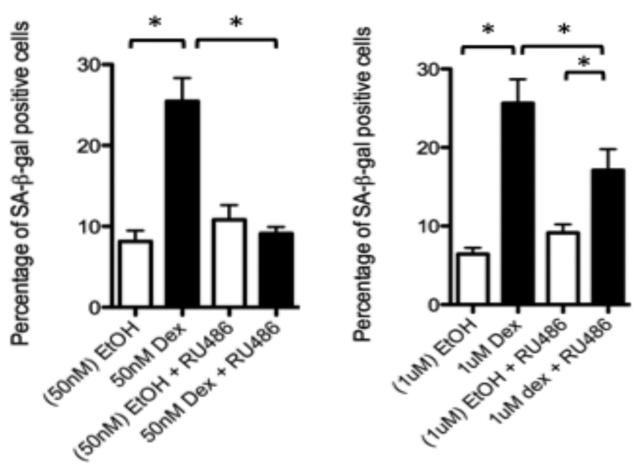

$\mathrm{F}$
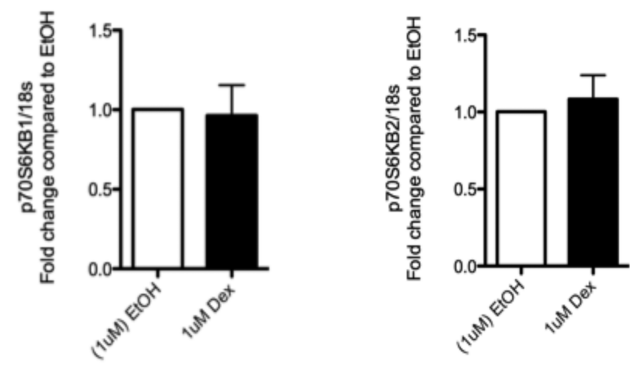

G

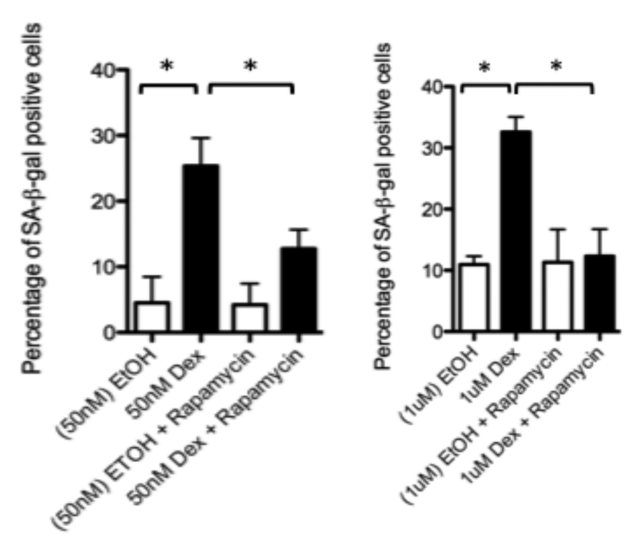


mTOR remains active in glucocorticoid-treated tenocytes

We found no difference in levels of phosphorylated p70 S6K (figure 1E) or in RNA levels of either of the p70S6K subunits (figure $1 \mathrm{~F}$ ) between dex-treated tenocytes and controls. To confirm that mTOR was active following dex treatment, we co-treated cells with dex and the mTOR inhibitor rapamycin and found this led to a significant reduction in SA- $\beta$-gal-positive cells (figure $1 \mathrm{G}$ ).

\section{Glucocorticoids activate the p53 pathway}

There are two major pathways leading to induction of cellular senescence: the p38 mitogen activated protein kinase (MAPK)/ p $16^{\text {INK4a }}$ pathway and the $\mathrm{p} 53 / \mathrm{p} 21^{\text {cip }}$ pathway. ${ }^{29}$ We found no evidence of an increase in levels of phosphorylated p38 MAPK or $\mathrm{p} 16^{\mathrm{INK} 4 \mathrm{a}}$ in dexamethasone-treated tenocytes compared with controls (figure 2A). In contrast, levels of acetylated p53 (figure 2B) (but not phosphorylated p53) (see online supplementary figure S3) were higher in glucocorticoid-treated tenocytes compared with controls. While there were no significant differences in p53 RNA levels, RNA levels of $\mathrm{p} 21^{\text {cip/waf1 }}$, a pro-senescence modulator of p53 activity, were markedly elevated post-glucocorticoid treatment (figure 2C).

Figure 2 Glucocorticoids activate p53 signalling in tenocytes. Western blots showing levels of $(A)$

phosphorylated and total p38 MAPK and p16 ${ }^{\mathrm{INK} 4 a}$ and (B) acetylated (K320) and total p53 and p21 $1^{\text {cip/waf1 }}$ in lysates of primary human tenocytes following treatment with $1 \mu \mathrm{M}$ dexamethasone or carrier (ethanol) for $48 \mathrm{~h}$. Western blots were conducted on protein lysates from cells obtained from three different tissue donors. Blots shown are representative of all experiments. (C) No significant differences in RNA levels of p53 were apparent in tenocytes treated with $1 \mu \mathrm{M}$ dexamethasone compared with ethanol-treated controls. However, RNA levels of p21 were significantly greater in tenocytes treated with dexamethasone compared with ethanol-treated controls. (D) The percentage of SA- $\beta$ gal-positive cells was significantly lower in cells treated with dexamethasone ( $50 \mathrm{nM}$ or $1 \mu \mathrm{M}$ ) and the p53 inhibitor pifithrin- $\alpha$ (PFT- $\alpha, 100 \mathrm{nM}$ ) compared with cells treated with dexamethasone alone, $n=3$. (E) Western blot demonstrating the effectiveness of p53 knockdown obtained using RNAi in primary human tenocytes. (F) The percentage of SA- $\beta$ gal-positive cells was significantly lower in tenocytes in which p53 expression was knocked down (sip53) compared with dexamethasone-treated controls (siCntrl) $(n=3)$. Statistically significant differences between ethanol (EtOH) and dexamethasone (Dex) and between dexamethasone with and without the p53 inhibitor (PFT- $\alpha$ ) or with and without p53 siRNA (sip53) are indicated by * $(p<0.05)$.

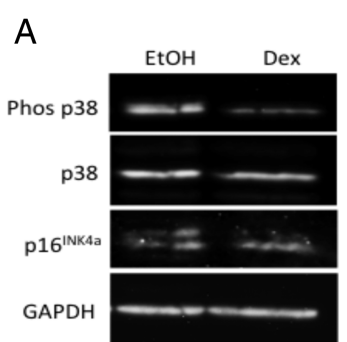

B

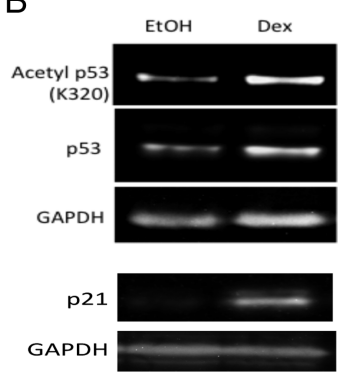

D

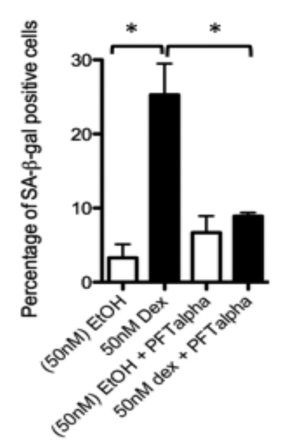

$\mathrm{F}$

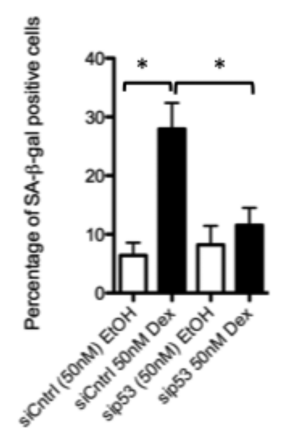

To confirm the involvement of p53 activity in glucocorticoidinduced senescence, we co-treated tenocytes with dexamethasone and the p53 inhibitor pifithrin- $\alpha$ (PFT- $\alpha, 100 \mathrm{nM})$ for $72 \mathrm{~h}$. We found the number of SA- $\beta$-gal-positive cells was significantly lower in dexamethasone+inhibitor-treated cells compared with those treated with dexamethasone alone (figure 2D). Similarly, we found the number of SA- $\beta$-gal-positive cells was significantly lower in dexamethasone-treated cells in which p53 expression was knocked down using RNAi compared with dexamethasone-treated cells transfected with a non-targeting siRNA control (figure 2E, F).

\section{Glucocorticoids inhibit expression of sirtuin 1}

Reduced levels of the p53 deacetylase sirtuin 1 (sirt1) are often associated with $\mathrm{p} 53$-mediated senescence. ${ }^{30}$ We found a small but significant reduction in RNA levels of sirt1, but a marked reduction in protein levels in glucocorticoid-treated cells compared with carrier-treated controls (figure 3A,B). To determine whether sirt1 is involved in glucocorticoid-induced senescence, we overexpressed sirt 1 in tenocytes using adenoviral-mediated gene delivery. We found the percentage of SA- $\beta$-gal-positive cells was significantly lower in dexamethasone-treated tenocytes infected with a sirt1bearing adenoviral vector (adSirt1) compared with

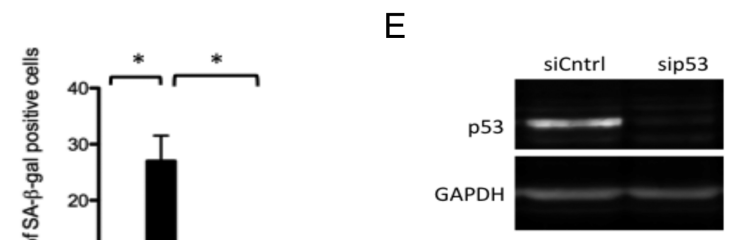


dexamethasone-treated tenocytes infected with a GFP-bearing control adenoviral vector (adGFP) (figure 3C,D).

\section{Prevention of the glucocorticoid-mediated inhibition of} sirtuin 1 expression protects against senescence induction

sirt 1 is activated by caloric restriction and by the plant-derived phenol resveratrol. To simulate caloric restriction in vitro, we cultured cells in low $(2 \mathrm{mM})$ glucose media. We found protein levels of sirt1 were higher, and levels of acetylated p 53 lower, in tenocytes co-treated with dexamethasone and resveratrol or in dexamethasone-treated tenocytes grown in low glucose media compared with cells treated with dexamethasone alone and grown in standard (17.5 $\mathrm{mM}$ glucose) culture media (figure 4A). The number of SA- $\beta$-gal-positive cells was also significantly lower in cells co-treated with dexamethasone and resveratrol or treated with dexamethasone in low glucose media compared with cells treated with dexamethasone alone indicating these treatments can protect against glucocorticoid-induced senescence in vitro (figure 4B, C). In order to confirm the involvement of sirt1 activity in the protective effect of these two treatments against glucocorticoid-induced senescence, we used a specific sirt1 inhibitor (100 nM EX527). The percentage of SA- $\beta$-gal-positive cells was significantly higher in dexamethasone-treated cells co-treated with the sirt1 inhibitor
Figure 3 Glucocorticoids inhibit sirtuin 1 expression in tenocytes. Both (A) RNA (as measured by real-time qRT-PCR $7 \mathrm{~h}$ post-treatment, $\mathrm{n}=3$ ) and (B) protein (as determined by western blotting) levels of sirtuin 1 were reduced in dexamethasone-treated cells compared with carrier (ethanol)-treated controls. Western blots were conducted on protein lysates from cells obtained from three different tissue donors. Blot shown is representative of all experiments. (C) The percentage of SA- $\beta$ gal-positive cells was significantly lower in dexamethasone-treated tenocytes infected with an adenoviral vector containing a sirtuin 1 overexpression construct (adSirt1) compared with dexamethasone-treated tenocytes infected with a control adenoviral vector expressing GFP (adGFP). (D) Western blot demonstrating the effectiveness of the adSirt1 virus to prevent dexamethasone-induced inhibition of sirtuin 1 expression. Statistically significant differences between ethanol (EtOH) and dexamethasone (Dex) and between dexamethasone with and without sirt1 overexpression (adSirt1) are indicated by * $(p<0.05)$.
A

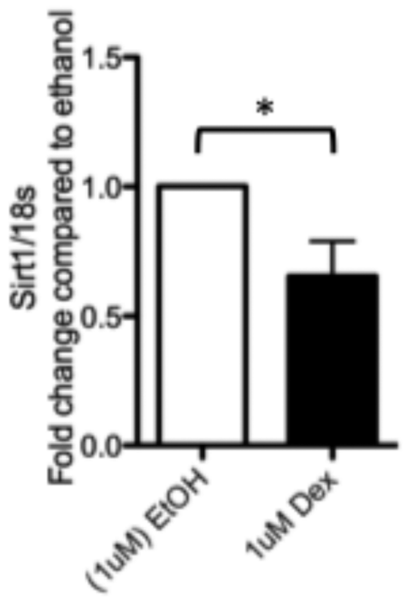

C

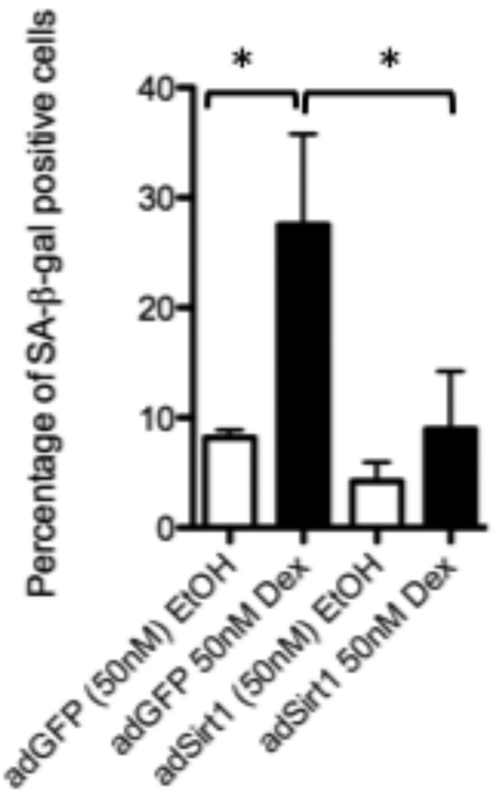

B

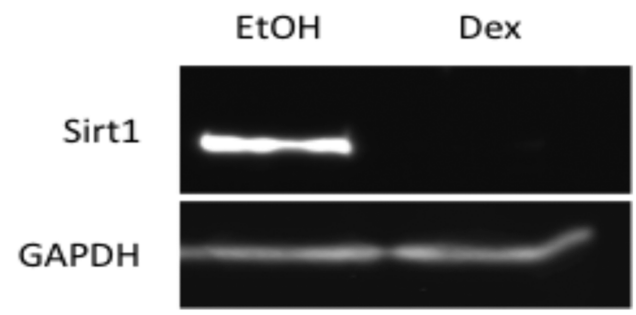

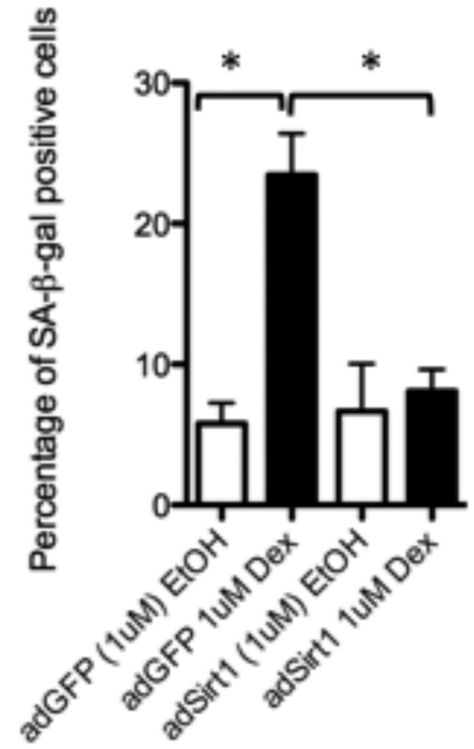

D

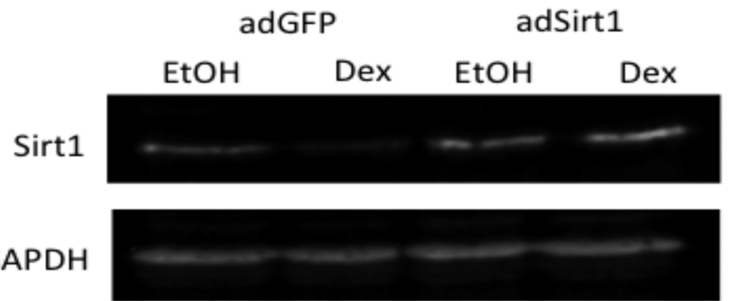


and cultured in low glucose $(2 \mathrm{mM})$ media (figure 4D) or treated with resveratrol $(30 \mu \mathrm{M})$ (figure $4 \mathrm{E}$ ) compared with cells grown under the same conditions without the sirt1 inhibitor.

\section{Evidence of p53 pathway activation following local injection} of glucocorticoids in patients with rotator cuff disease

Tendon biopsies were taken from five patients with rotator cuff disease immediately preceding and 7 weeks following glucocorticoid injection. Using immunohistochemistry, we found the percentage of $\mathrm{p} 53$-positive cells was significantly higher $(\mathrm{p}=0.03)$ post-injection compared with pre-injection (figure $5 \mathrm{~A})$.
The percentage of $\mathrm{p} 21$-positive cells also tended to be higher post-injection $(p=0.06)$ (figure $5 \mathrm{~B})$. There was no significant difference in the degree of tendinopathy as assessed by Bonar score between pre-injection and post-injection tissue biopsies (see online supplementary figure S4).

\section{Glucocorticoids also induce senescent-like changes in primary human chondrocytes and osteoblasts}

To determine whether other cell types also undergo senescencelike changes following dexamethasone treatment, we treated primary human chondrocytes and primary human osteoblasts
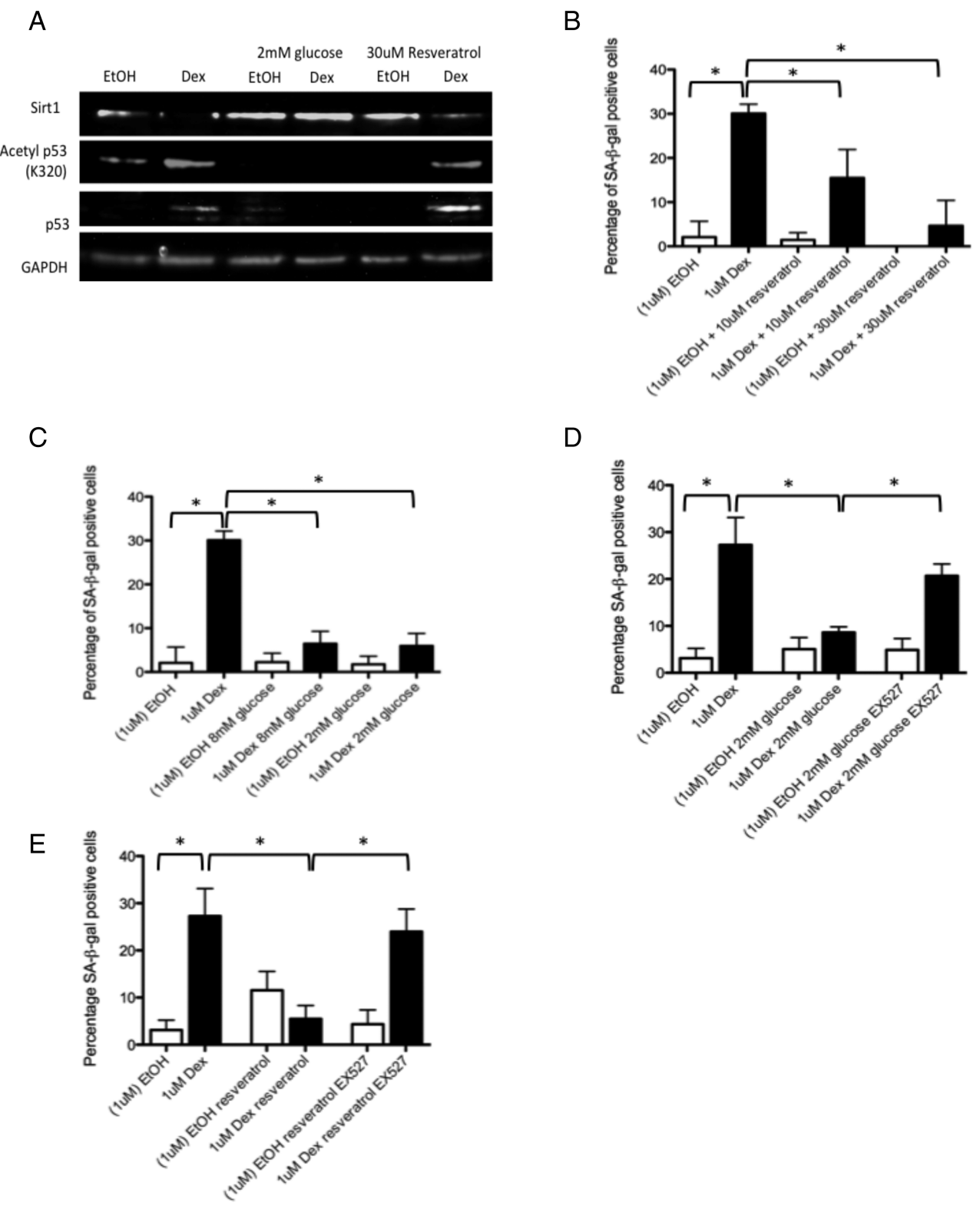

Figure 4 Protective effect of low glucose, low serum and resveratrol on glucocorticoid-induced senescence (A) Protein levels of sirtuin 1 were higher in tenocytes treated with dexamethasone $(1 \mu \mathrm{M})$ and cultured in low glucose $(2 \mathrm{mM})$ media or co-treated with resveratrol $(30 \mu \mathrm{M})$ compared with tenocytes treated with dexamethasone and cultured in standard growth media (17.5 mM glucose). Levels of acetylated p53 (lys320) were lower in dexamethasone-treated cells cultured in low glucose $(2 \mathrm{mM})$ media or co-treated with resveratrol compared with tenocytes treated with dexamethasone and cultured in standard growth media (17.5 mM glucose). Western blots were conducted on protein lysates from cells obtained from three different tissue donors. Blots shown are representative of all three experiments. The percentage of SA- $\beta$ gal-positive cells was significantly lower in dexamethasone-treated cells (B) co-treated with resveratrol $(10$ or $30 \mu \mathrm{M})$ or $(\mathrm{C})$ cultured in low glucose $(2$ or 8 mM) media; however, no significant difference in the percentage of SA- $\beta$ gal-positive cells was observed following addition of EX527, a sirtuin 1 inhibitor, in dexamethasone-treated cells $(1 \mu \mathrm{M})$ cultured in (D) $2 \mathrm{mM}$ glucose or (E) co-treated with resveratrol $(30 \mu \mathrm{M})$ compared with cells treated with dexamethasone alone and cultured in standard $(17.5 \mathrm{mM})$ growth media $(\mathrm{n}=3)$. Statistically significant differences between ethanol $(\mathrm{EtOH})$ and dexamethasone (Dex) and between dexamethasone with and without either resveratrol or $2 \mathrm{mM}$ glucose are indicated by * $(p<0.05)$. 
with dexamethasone $(1 \mu \mathrm{M})$ for $72 \mathrm{~h}$. We found the percentage of SA- $\beta$-gal-positive cells was significantly higher in both cell types following dexamethasone treatment (see online supplementary figure S5).

\section{DISCUSSION}

In the present study, we have demonstrated that glucocorticoids induce senescence in tenocytes in vitro by the $\mathrm{p} 53 / \mathrm{p} 21$ pathway. We have also shown that the $\mathrm{p} 53 / \mathrm{p} 21$ pathway is upregulated in tendon tissue 7 weeks after local injection of glucocorticoids suggesting that glucocorticoids also induce senescence in vivo. These findings represent a previously undescribed mechanism of action of glucocorticoids likely to have important clinical implications.

We, and others, have previously reported that glucocorticoid treatment of tenocytes leads to inhibition of cell proliferation $^{79}{ }^{10}$ but whether this results in cell quiescence or senescence was unknown. One of the critical features distinguishing a senescent cell from a quiescent cell is that mTOR remains active in the former. We found no change in levels of phosphorylated p70 S6kinase, a substrate of the mTORC1 enzyme, following glucocorticoid treatment of tenocytes in situ suggesting mTOR remained active in glucocorticoid-treated cells. Consistent with this finding, SA- $\beta$-gal activity was greater in glucocorticoidtreated tenocytes compared with controls. Inhibition of mTOR with rapamycin markedly reduced the number of SA- $\beta$-gal-positive cells following glucocorticoid treatment. Taken together, these results confirm that glucocorticoids induce senescence in primary human tenocytes.

Circulating endogenous glucocorticoids are mostly protein bound and as such are inactive. Local injection of glucocorticoids results in a large influx of unbound and therefore active glucocorticoid into the joint. Similarly, almost all of the glucocorticoids administered in our in vitro experiments would have been 'free', that is, available for receptor binding. In the present study, we saw no evidence of a dose effect of glucocorticoids on senescence induction. It is likely that the large excess of unbound glucocorticoid provided even by our lowest glucocorticoid dose fully occupied receptor sites, resulting in maximal receptor activation.
Figure 5 The p53/p21 pathway is activated in vivo following local injection with glucocorticoids. Tendon biopsy samples were taken from five patients with rotator cuff tendinopathy immediately prior to subacromial injection with the synthetic glucocorticoid, Depo-Medrone, and compared with subsequent biopsy specimens taken from the same patients 7 weeks following glucocorticoid injection. Using immunohistochemistry, (A) the percentage of $\mathrm{p53}$-positive cells was significantly greater in tissue samples post-injection compared with pre-injection. (B) A non-statistically significant increase in the percentage of p21-positive cells was observed in the post-injection tissue samples compared with pre-injection tissue samples. Representative

photomicrographs are shown for tissue samples from two patients.

p53-positive cells or p21-positive cells were detected using horseradish peroxidase-conjugated secondary antibodies and diaminobenzadine. Positive cells appear brown. Tissue was counterstained with haematoxylin (blue) to allow quantification of total cell numbers. Statistically significant differences are indicated by * $(p<0.05)$.
A
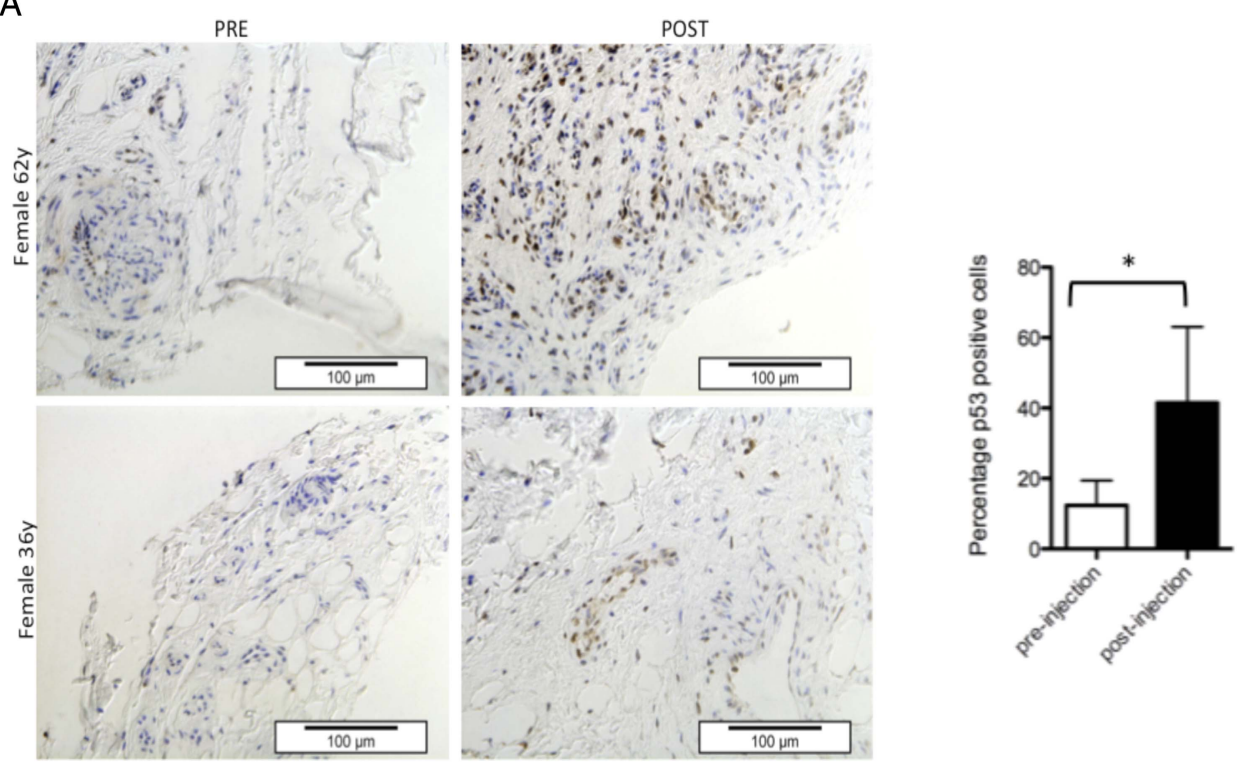

B

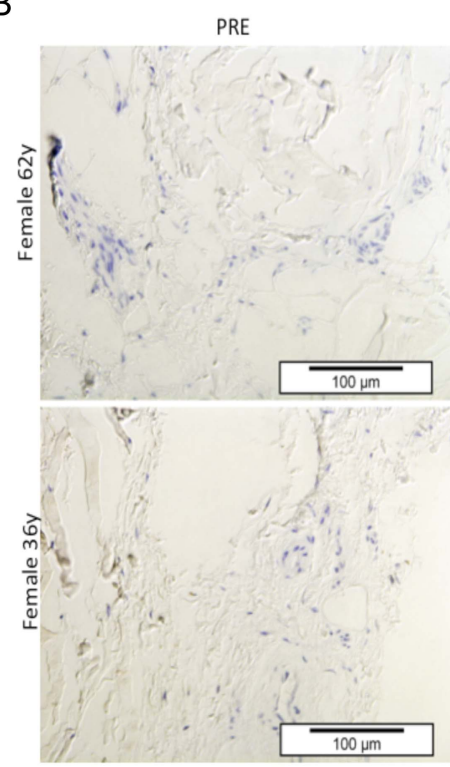


There are two major signalling pathways which can lead to induction of cellular senescence: the p38 MAPK/p $16^{\mathrm{INK} 4 \mathrm{a}}$ pathway and the $\mathrm{p} 53 / \mathrm{p} 21^{\text {cip }}$ pathway (reviewed in ref. ${ }^{29}$ ). We found no evidence of activation of the p38 MAPK/p $16^{\text {INK4a }}$ pathway in glucocorticoid-treated tenocytes. In contrast, we found levels of acetylated p53 were higher in glucocorticoidtreated tenocytes compared with carrier-treated controls indicating glucocorticoids activate p53 signalling in tenocytes. Consistent with our results, Crochemore $e t a l^{31}$ found that GR activation enhanced p53 activity in neural cells. Acetylation of p53 on K320 promotes the transcription of pro-survival (as opposed to pro-apoptosis) p53-regulated genes. It also stabilises the $\mathrm{p} 53$ protein by preventing its nuclear export. ${ }^{32}$ This stabilisation of p53 may be responsible for the increase in total p53 protein levels also observed in glucocorticoid-treated cells in the present study.

p53 activation can lead to either promotion of apoptosis or induction of senescence. p $21^{\text {cip }}$ is a cell cycle controller which is critical for determining the outcome of p53 activation. p21 cip induces cell cycle arrest and inhibits the pro-apoptotic activity of p53 channelling p53 activity towards senescence induction. ${ }^{33}$ Glucocorticoids have previously been shown to directly stimulate $\mathrm{p} 21^{\mathrm{cip}}$ transcription. ${ }^{34}$ In the present study, we observed increased $\mathrm{p} 21^{\text {cip }}$ expression following glucocorticoid treatment of tenocytes indicating the pro-senescence functionality of p53 was activated by glucocorticoid treatment. Confirming the involvement of p53 signalling in glucocorticoid-induced senescence, we found inhibiting p53 activity or knockdown of p53 expression in glucocorticoid-treated tenocytes resulted in significantly fewer SA- $\beta$-gal-positive cells.

p53 is deacetylated by sirt $1 .{ }^{35}{ }^{36}$ Reduced sirt 1 levels and/or activity are frequently associated with increased p53 acetylation and increased p53-mediated senescence. ${ }^{37} 38$ We found glucocorticoid treatment resulted in a significant reduction in both RNA and protein levels of sirt1 in vitro. To our knowledge, this is the first report describing glucocorticoid-mediated regulation of sirt1 expression. Caloric restriction and the plant-derived phenol resveratrol increase both the expression ${ }^{39}$ and the activity of sirt $1 .^{40-42}$ In the present study, we found lowering the glucose content of the cell culture medium (to emulate caloric restriction) or treatment with resveratrol protected against the glucocorticoid-induced reduction in sirt1 levels, partially ameliorated the increase in acetylated p53 levels and led to significantly fewer SA- $\beta$-gal-positive cells. The inhibitory effect of low glucose and resveratrol on glucocorticoid-induced senescence was abolished by the addition of a sirt 1 inhibitor demonstrating that the activation of sirt 1 was largely responsible for their protective effect. In concurrence with these results, we found overexpression of sirt 1 in tenocytes using adenoviral-mediated gene transduction also protected against glucocorticoid-induced senescence.

To determine whether glucocorticoid treatment also induces senescence in patients, we compared supraspinatus biopsies taken before and after subacromial glucocorticoid injection in five patients with rotator cuff tendinopathy. Using this set of biopsy specimens, we have previously found that the percentage of cells positive for the proliferation marker mib-1 was significantly lower, but the percentage of apoptotic cells was not significantly different in post-injection biopsies compared with pre-injection biopsies (manuscript in preparation). In the present study, we found the percentage of p53-positive cells was significantly higher in post-injection biopsies compared with pre-injection biopsies $(\mathrm{p}=0.03)$. The percentage of $\mathrm{p} 21$-positive cells also tended to be higher post-injection compared with pre- injection $(p=0.06)$. These data provide compelling evidence that the $\mathrm{p} 53 / \mathrm{p} 21$ pathway is upregulated following glucocorticoid injection in patients suggesting that glucocorticoids also induce senescence in vivo.

Synthetic glucocorticoids are extensively used to treat painful and inflammatory conditions in a variety of tissues. In vitro, we found the percentage of SA- $\beta$-gal-positive cells was also higher following glucocorticoid treatment of primary human osteoblasts and chondrocytes suggesting glucocorticoids may induce senescence in a range of different cell types.

The loss of normal functionality associated with senescence has been implicated in age-related disease and degeneration. ${ }^{43} 44$ Given the apparent irreversible nature of senescence, glucocorticoid-induced senescence is likely to have long-term detrimental consequences for tissue. That we observed a marked increase in p53 expression 7 weeks following glucocorticoid injection supports the notion that the effects of local glucocorticoid injection on tendon tissue are not transient. Glucocorticoid-induced senescence may exacerbate the underlying tissue pathology responsible for the pain for which the glucocorticoids are prescribed to treat.

Acknowledgements The authors gratefully acknowledge Mr Chethan Jayadev, Mr Ben Davies, Professor Andrew Price and the Oxford Musculoskeletal Biobank (OMB), University of Oxford, UK for collection of human hamstring, cartilage and bone tissue. Sincere thanks to Dr Benjamin Dean and Dr Sarah Franklin for tissue sectioning and help with immunohistochemistry. To research nurses, Mrs Kim Wheway and Mrs Bridget Watkins for help with collection of biopsies pre-glucocorticoid and post-glucocorticoid injection. Special thanks also to the patients who donated their time and tissue to make this study possible.

Contributors RP was involved in conception and design of the study, acquisition, analysis and interpretation of data and writing of the manuscript. AW was involved in data acquisition and writing of the manuscript. RM was involved in conception and design of the biopsy study, collection of biopsy specimens and writing of the manuscript. SS was involved in data acquisition and revision of the manuscript. AC was involved in conception and design of the biopsy study, biopsy collection and critical review of the manuscript. PH was involved in study design and review of the manuscript.

Funding This study was funded by Arthritis Research UK (grant no. 19482) and the NIHR Oxford Musculoskeletal Biomedical Research Unit. The extent of involvement of both sponsors in this study was purely financial.

\section{Competing interests None.}

Ethics approval Oxfordshire REC B.

Provenance and peer review Not commissioned; externally peer reviewed.

Open Access This is an Open Access article distributed in accordance with the Creative Commons Attribution Non Commercial (CC BY-NC 3.0) license, which permits others to distribute, remix, adapt, build upon this work non-commercially, and license their derivative works on different terms, provided the original work is properly cited and the use is non-commercial. See: http://creativecommons.org/ licenses/by-nc/3.0/

\section{REFERENCES}

1 NHS Business Services Authority. Electronic Prescribing \& Financial Information for Practices (ePFIP). http://wwwnhsbsanhsuk/PrescriptionServices/963aspx, 2009

2 Garbe E, LeLorier J, Boivin JF, et al. Inhaled and nasal glucocorticoids and the risks of ocular hypertension or open-angle glaucoma. JAMA 1997;277:722-7.

3 Oikarinen A, Autio P. New aspects of the mechanism of corticosteroid-induced dermal atrophy. Clin Exper Dermatol 1991;16:416-19.

4 Butler RC, Davie MWJ, Worsfold M, et al. Bone-mineral content in patients with rheumatoid-arthritis—relationship to low-dose steroid-therapy. Br J Rheumatol 1991;30:86-90

5 Ford LT, Debender J. Tendon-rupture after local steroid injection. South Med J 1979;72:827-30.

6 Nichols AW. Complications associated with the use of corticosteroids in the treatment of athletic injuries. Clin J Sport Med 2005;15:370-5.

7 Scutt N, Rolf CG, Scutt A. Glucocorticoids inhibit tenocyte proliferation and Tendon progenitor cell recruitment. J Orthop Res 2006;24:173-82.

8 Wong MWN, Lui WT, Fu SC, et al. The effect of glucocorticoids on tendon cell viability in human tendon explants. Acta Orthop 2009;80:363-7.

9 Torricelli P, Fini M, Giavaresi G, et al. Effects of systemic glucocorticoid administration on tenocytes. Biomed Pharmacother 2006;60:380-5. 
10 Poulsen R, Carr A, Hulley P. Protection against glucocorticoid-induced damage in human tenocytes by modulation of ERK, Akt and forkhead signalling. Endocrinology 2011:152:503-14.

11 Hampel B, Fortschegger K, Ressler, et al. Increased expression of extracellular proteins as a hallmark of human endothelial cell in vitro senescence. Exp Gerontol 2006:41:474-81.

12 Millis AJT, Hoyle M, Mccue HM, et al. Differential expression of metalloproteinase and tissue inhibitor of metalloproteinase genes in aged human fibroblasts. Exp Cell Res 1992;201:373-9.

13 Abate M, Gravare-Silbernagel K, Siljeholm C, et al. Pathogenesis of tendinopathies: inflammation or degeneration? Arthritis Res Ther 2009;11:235-49.

14 Millar NL, Wei AQ, Molloy TJ, et al. Cytokines and apoptosis in supraspinatus tendinopathy. Journal of Bone and Joint Surgery-British Volume 2009;91B:417-24.

15 Demidenko ZN, Blagosklonny MV. Growth stimulation leads to cellular senescence when the cell cycle is blocked. Cell Cycle 2008;7:3355-61.

16 Korotchkina LG, Leontieva OV, Bukreeva El, et al. The choice between p53-induced senescence and quiescence is determined in part by the mTOR pathway. Aging 2010:2:344-52.

17 Park IH, Bachmann R, Shirazi $H$, et al. Regulation of ribosomal S6 kinase 2 by mammalian target of rapamycin. J Biol Chem 2002;277:31423-9.

18 Kubica N, Crispino JL, Gallagher JW, et al. Activation of the mammalian target of rapamycin complex 1 is both necessary and sufficient to stimulate eukaryotic initiation factor 2Bvarepsilon mRNA translation and protein synthesis. Int J Biochem Cell Biol 2008:40:2522-33.

19 Kubica N, Bolster DR, Farrell PA, et al. Resistance exercise increases muscle protein synthesis and translation of eukaryotic initiation factor 2Bepsilon mRNA in a mammalian target of rapamycin-dependent manner. J Biol Chem 2005;280:7570-80.

20 Blagosklonny MV. Cell senescence: hypertrophic arrest beyond the restriction point. J Cell Physiol 2006;209:592-7.

21 Blagosklonny MV. Cell cycle arrest is not senescence. Aging 2011;3:94-101.

22 Dimri GP, Lee $\mathrm{X}$, Basile G, et al. A biomarker that identifies senescent human cells in culture and in aging skin in vivo. Proc Nat Acad Sci. 1995;92:9363-7.

23 Livak KJ, Schmittgen TD. Analysis of relative gene expression data using real-time quantitative PCR and the 2(-Delta Delta C(T)) Method. Methods 2001;25:402-8.

24 Kessel L, Watson $M$. The painful arc syndrome. Clinical classification as a guide to management. J Bone Joint Surg 1977;59:166-72.

25 Hawkins R, Kennedy J. Impingement syndrome in athletes. Am J Sports Med 1980;8:151-8.

26 Jobe $F$, Moynes D. Delineation of diagnostic criteria and a rehabilitation program for rotator cuff injuries. Am J Sports Med 1982;10:336-9.

27 Cook J, Feller J, Bonar $S$, et al. Abnormal tenocyte morphology is more prevalent than collagen disruption in asymptomatic athletes' patellar tendons. J Orthop Res 2004;22:334-8.

28 Czock D, Keller F, Rasche F, et al. Pharmacokinetics and pharmacodynamics of systemically administered glucocorticoids. Clin Pharmacokinet 2005;44:61-98.
29 Muller M. Cellular Senescence: Molecular Mechanisms, In Vivo Significance, and Redox Considerations. Antioxid Redox Signal 2009;11:59-98.

30 Langley $\mathrm{E}$, Pearson $\mathrm{M}$, Faretta $\mathrm{M}$, et al. Human SIR2 deacetylates p53 and antagonises PML/p53-induced cellular senescence. Embo J 2002; 21:2383-96.

31 Crochemore C, Michaelidis TM, Fischer D, et al. Enhancement of p53 activity and inhibition of neural cell proliferation by glucocorticoid receptor activation. FASEB $J$ 2002:16.

32 Knights CD, Catania J, Di Giovanni S, et al. Distinct p53 acetylation cassettes differentially influence gene-expression patterns and cell fate. J Cell Biol 2006;173:533-44.

33 Fang $\mathrm{L}$, Igarashi $\mathrm{M}$, Leung J, et al. p21Waf1/Cip1/Sdi1 induces permanent growth arrest with markers of replicative senescence in human tumor cells lacking functional p53. Oncogene 1999:18:2789-97.

34 Cha H, Cram E, Wang E, et al. Glucocorticoids Stimulate p21 Gene Expression by Targeting Multiple Transcriptional Elements within a Steroid Responsive Region of the p21waf1/cip1 Promoter in Rat Hepatoma Cells. J Biol Chem 1998:273:1998-2007.

35 Luo JY, Nikolaev AY, Imai S, et al. Negative control of p53 by Sir2 alpha promotes cell survival under stress. Cell 2001;107:137-48.

36 Vaziri H, Dessain SK, Eagon EN, et al. hSIR2(SIRT1) functions as an NAD-dependent p53 deacetylase. Cell 2001;107:149-59.

37 Ota $\mathrm{H}$, Akishita M, Masato $\mathrm{E}$, et al. Sirt1 modulates premature senescence-like phenotype in human endothelial cells. Journal of Molecular and Cellular Cardiology 2007:43:571-9.

38 Ota $\mathrm{H}$, Kaneki M, lijima K, et al. Inhibition of Sirt1, a human homolog of yeast longevity gene, Sir2, induces senescence-like phenotype in human endothelial cells. Circulation 2005;112:U351-U351.

39 Nemoto S, Fergusson MM, Finkel T. Nutrient availability regulates SIRT1 through a forkhead-dependent pathway. Science 2004:306:2105-8.

40 Evans C, Bogan K, Song P, et al. NAD+ metabolite levels as a function of vitamins and calorie restriction: evidence for different mechanisms of longevity. BMC Chem Biol 2010;10:1-10.

41 Howitz KT, Bitterman KJ, Cohen HY, et al. Small molecule activators of sirtuins extend Saccharomyces cerevisiae lifespan. Nature 2003;425:191-6.

42 Wang J, Zhang $Y$, Tang $L$, et al. Protective effects of resveratrol through the up-regulation of SIRT1 expression in the mutant hSOD1-G93A-bearing motor neuron-like cell culture model of amyotrophic lateral sclerosis. Neurosci Lett 2011:503:250-5.

43 Heathfield S, Le Maitre C, Hoyland J. Caveolin-1 expression and stress-induced premature senescence in human invertebral disc degeneration. Arthritis Res Ther 2008;10:doi:10.1186/ar2468

44 Koziowski M. RPE cell senescence: A key contributor to age-related macular degeneration. Med Hypotheses 2012;78:505-10. 